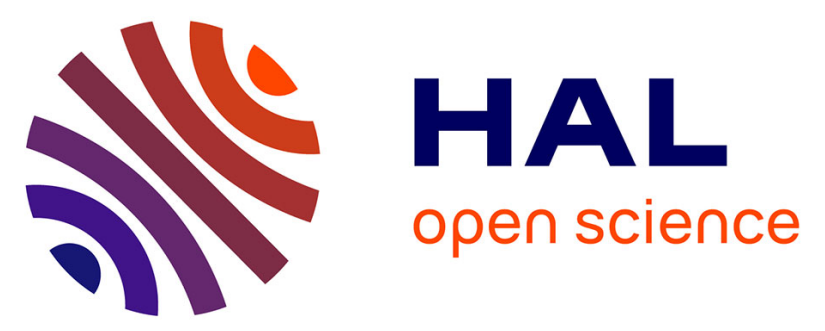

\title{
Crystallization profile and morphological study of SAPO-5 templated by imidazolium cations of different functional groups
}

\author{
Ismail Alhassan Auwal, Svetlana Mintova, Tau Chuan Ling, Fitri
}

Khoerunnisa, Ka-Lun Wong, Eng-Poh Ng

\section{To cite this version:}

Ismail Alhassan Auwal, Svetlana Mintova, Tau Chuan Ling, Fitri Khoerunnisa, Ka-Lun Wong, et al.. Crystallization profile and morphological study of SAPO-5 templated by imidazolium cations of different functional groups. Microporous and Mesoporous Materials, 2020, 308, pp.110514. 10.1016/j.micromeso.2020.110514 . hal-02967627

\section{HAL Id: hal-02967627 \\ https://hal.science/hal-02967627}

Submitted on 26 Nov 2020

HAL is a multi-disciplinary open access archive for the deposit and dissemination of scientific research documents, whether they are published or not. The documents may come from teaching and research institutions in France or abroad, or from public or private research centers.
L'archive ouverte pluridisciplinaire $\mathbf{H A L}$, est destinée au dépôt et à la diffusion de documents scientifiques de niveau recherche, publiés ou non, émanant des établissements d'enseignement et de recherche français ou étrangers, des laboratoires publics ou privés. 


\title{
Crystallization profile and morphological study of SAPO-5 templated
}

\section{by imidazolium cations of different functional groups}

Ismail Alhassan Auwal, ${ }^{1,2}$ Svetlana Mintova, ${ }^{3}$ Tau Chuan Ling, ${ }^{4}$ Ka-Lun Wong,,${ }^{5 *}$ Eng-Poh Ng ${ }^{1 *}$

${ }^{1}$ School of Chemical Sciences, Universiti Sains Malaysia, 11800 USM, Penang, Malaysia.

${ }^{2}$ Chemistry Department, Faculty of Natural and Applied Sciences, Sule Lamido University, PMB 048 Kafin Hausa, Jigawa State, Nigeria.

${ }^{3}$ Normandie Univ., ENSICAEN, UNICAEN, CNRS, Laboratoire Catalyse et Spectrochimie, 14000 Caen, France.

${ }^{4}$ Institute of Biological Sciences, Faculty of Science, University of Malaya, 50603 Kuala Lumpur, Malaysia.

${ }^{5}$ School of Energy and Chemical Engineering, Xiamen University Malaysia, 43900 Sepang, Selangor, Malaysia.

${ }^{6}$ College of Chemistry and Chemical Engineering, Xiamen University, Xiamen 361005, China.

*Corresponding authors: epng@usm.my (EPN); kalun.wong@xmu.edu.my (KLW)

\begin{abstract}
1-Propyl-2,3-dimethyl-1H-imidazol-3-ium $\quad\left([\mathrm{pmIm}]^{+}\right), \quad 3$-(2,3-dihydroxypropyl)-1,2-dimethyl-1Himidazol-3-ium $\left([\mathrm{dhmIm}]^{+}\right)$and 1-benzyl-2,3-dimethyl-1H-imidazol-3-ium $\left([\mathrm{bzmIm}]^{+}\right)$cations were used as organic templates for crystallizing SAPO-5 with AFI microporous framework. The physicochemical influences of the functional groups of the templates (e.g. polarity/hydrophilicity, molecular dimension and size) on SAPO-5 formation were investigated. The results revealed that the crystallization rate, crystallinity and solid yield could be enhanced by increasing the polarity/hydrophilicity of the templates. In addition, the isomorphous Si substitution, morphology and particle size of the resulted SAPO-5 were also affected by the substituted groups of the imidazolium
\end{abstract}


templates. The thermogravimetry results further supported that the selection of organic template with proper polarity/hydrophilicity could be crucial to obtain SAPO-5 with high porosity $\left(S_{\mathrm{BET}}, \mathrm{S}_{\mathrm{mic}}, \mathrm{S}_{\mathrm{ext}}\right.$ and $\left.\mathrm{V}_{\text {total }}\right)$ as high polar/hydrophilic template exhibited stronger chemical interaction with the AFI framework during template removal process via calcination process.

Keywords: SAPO-5; Microporous material; Hydrothermal synthesis; Imidazolium template; Functional group; Polarity.

\section{Introduction}

The aluminophosphate (AlPO- $n$ ) and silicoaluminophosphate (SAPO- $n$ ) crystalline solids are zeolite-like materials with microporous framework that have been widely used in catalysis, adsorption and separation [1-3]. Recently, they have found new applications in nanotechnology, biotechnology and medical diagnosing [4-6]. A rational synthesis of these microporous materials with unique structures and properties is hence highly desired, but remains challenging.

Recently, several works have been devoted to understand the crystallization behavior of the AlPO- $n$ and SAPO- $n$ materials $[7,8]$. Nevertheless, the crystal growth on a microscopic level are still not well-understood because the formation of these crystalline solids is depending on many synthesis parameters which include the molar ratio of reagents, solvent content, hydrothermal temperature, crystallization and aging times, the selection of templates, and so on [7-9]. Certainly, organic templates play a crucial role during the crystallization process by residing within the lattice of inorganic framework via non-chemical interaction, hence stabilizing the crystal structure and governing the morphology and porosity of the solid product [10]. In the synthesis of nanoporous materials, organic templates often serve as space fillers, structure directing agents, and/or true templating agents to facilitate the development of a specific porous framework [11, 12]. To date, a few groups of nitrogen- 
based organic substances like amines $[13,14]$, quartenary ammonium $[15,16]$, imidazolium $[17,18]$ and pyridinium [19] ions have been used as organic templates to prepare AlPO- $n$ and SAPO- $n$ materials.

SAPO-5 (with AFI framework) is a large-pore silicoaluminophosphate material. Its onedimensional cylindrical framework structure is made up of 12 -membered ring channels $(7.3 \AA)$ and constructed by $\mathrm{SiO}_{4}, \mathrm{AlO}_{4}$ and $\mathrm{PO}_{4}$ tetrahedrals forming a negative framework [20]. It is found that the morphology and size of SAPO-5 crystals are strongly depending on the synthesis conditions (synthesis recipe, temperature, time duration and heating technique) and properties of the organic templates (ionic charge, hydrophobicity/hydrophilicity, molecular shape and size). AFI framework structure can be templated using various types of organic templates such as triethylamine [21], tetraethylamonium hydroxide [22], tripropylamine (TPA) [23], tetramethylpyrazine and N',N',N',N'-tetramethyl-1,3propanediamine (TMPD) [24] and 1-ethyl-2,3-dimethyl imidazolium hydroxide [25]. By using different organic templates, SAPO-5 solids with rod, plate, barrel, prism, broom-like, flower-like, disclike, spheroidal shapes have been reported [26-30]. Imidazolium ionic liquids have been explored as recyclable and reusable synthesis mediums. Therefore, the influence of imidazolium-based templates bearing different functional groups on the formation of SAPO-5 is worth to be further explored.

In this study, microporous SAPO-5 materials have been synthesized under hydrothermal condition using imidazolium-based templates. Three imidazolium cations containing different functional groups and polarity/hydrophilicity, namely 1-propyl-2,3-dimethyl-1H-imidazol-3-ium $\left([\mathrm{pmIm}]^{+}\right), 3-\left(2,3-\right.$ dihydroxypropyl)-1,2-dimethyl-1H-imidazol-3-ium $\left([\mathrm{dhmIm}]^{+}\right)$and 1-benzyl-2,3dimethyl-1H-imidazol-3-ium $\left([\mathrm{bzmIm}]^{+}\right)$, have been synthesized in the laboratory before being characterized and used in the hydrothermal synthesis of SAPO-5. Then, the effects of these organic templates on the crystallization rate, degree of crystallinity, solid yield, morphology, crystallite size, elemental composition and porosity of the solid products are investigated by using various characterization techniques. 


\section{Experimental}

\subsection{Preparation of imidazolium templates}

\subsubsection{Preparation of imidazolium ionic salts}

1-Propyl-2,3-dimethyl-1H-imidazol-3-ium bromide ([pmIm]Br), 3-(2,3-dihydroxypropyl)-1,2dimethyl-1H-imidazol-3-ium 4-methylbenzenesulfonate ([dhmIm]OTs) and 1-benzyl-2,3-dimethyl1H-imidazol-3-ium chloride $([\mathrm{bzmIm}] \mathrm{Cl})$ were synthesized according to the procedures given in the Supplementary Information with slight modifications [7, 25, 31].

\subsubsection{Preparation of [pmIm]OH, [dhmIm]OH and [bzmIm]OH imidazolium hydroxide solutions}

1-Propyl-2,3-dimethyl-1H-imidazol-3-ium hydroxide ([pmIm]OH), 3-(2,3-dihydroxypropyl)1,2-dimethyl-1H-imidazol-3-ium hydroxide ([dhmIm]OH) and 1-benzyl-2,3-dimethyl-1H-imidazol-3ium hydroxide $([\mathrm{bzmIm}] \mathrm{OH})$ template solutions were prepared by ion exchanging the respective ionic salts with Amberlite ${ }^{\circledR}$ IRN-78 hydroxide ion-exchange resin (Sigma-Aldrich). The resulting solutions were separated from the resins by filtration and titrated with $0.1 \mathrm{M} \mathrm{HCl}$ solution to ensure $90 \% \mathrm{OH}^{-}$ ion exchange was achieved. The template solution in hydroxide form was then concentrated to 33.0 wt. $\%$ at $50{ }^{\circ} \mathrm{C}$ and stored at room temperature for further use.

\subsection{Crystallization of $S A P O-5$}

Typically, the SAPO-5 was synthesized as follows: Aluminum triisopropoxide (4.334 g, $\geq 99.99 \%$, Sigma-Aldrich), organic template solution (30.108 g for [pmIm]OH, $39.696 \mathrm{~g}$ for 
[bzmIm] $\mathrm{OH}$ or $39.221 \mathrm{~g}$ for $[\mathrm{dhmIm}] \mathrm{OH})$ and deionized water $(6.464 \mathrm{~g})$ were added and stirred in a polypropylene bottle for $35 \mathrm{~min}$. Then, $\mathrm{H}_{3} \mathrm{PO}_{4}(7.333 \mathrm{~g}, 85 \%$, Acros Organics) was added into the mixture in a dropwise manner for 15 min to produce a precursor mixture with a chemical composition of $1 \mathrm{Al}_{2} \mathrm{O}_{3}: 3 \mathrm{P}_{2} \mathrm{O}_{5}: 6 \mathrm{SDAOH}: 180 \mathrm{H}_{2} \mathrm{O}$ where $\mathrm{SDAOH}$ was the imidazolium hydroxide template used. The precursor mixture was transferred into an autoclave $(100 \mathrm{~mL})$ and heated at $180{ }^{\circ} \mathrm{C}$ for crystallization. After cooling, the solid product was washed with distilled water until $\mathrm{pH} 7$ and oven dried at $80{ }^{\circ} \mathrm{C}$. The final powder samples synthesized with $[\mathrm{pmIm}] \mathrm{OH},[\mathrm{bzmIm}] \mathrm{OH}$ and $[\mathrm{dhmIm}] \mathrm{OH}$ were labelled as pmIm- $n$, bzmIm- $n$ and dhmIm- $n$, respectively, where $n$ indicated the synthesis time in hour.

\subsection{Characterizations}

The XRD patterns of the samples were recorded with a Bruker-AXS D8 diffractometer $(\mathrm{Cu} \mathrm{K} \alpha$ radiation, $\lambda=1.5418 \AA$, $40 \mathrm{kV}$, scan speed of $0.2^{\circ} / \mathrm{min}$, step size of $0.02^{\circ}$ ). The crystallinity was estimated by comparing the intensity of the three most intense bands at $7.51^{\circ}, 19.92^{\circ}$ and $22.52^{\circ}$ of dhmIm-2.5 (as a reference of $100 \%$ crystallinity). The solid yield was determined in relation to the mass of the starting materials by using the following equation:

$$
\text { Solid yield }(\%)=\frac{\text { Mass of SAPO- } 5 \text { solid obtained }(\mathrm{g})}{\text { Initial mass of } 1 \mathrm{~mol} \text { of } \mathrm{P}_{2} \mathrm{O}_{5} \text { and } \mathrm{Al}_{2} \mathrm{O}_{3}(\mathrm{~g})} \times 100 \%
$$

The morphological properties of samples were characterized with a Leo Supra 50VP scanning electron microscope (FESEM) operated at $20 \mathrm{kV}$ and $10 \mu \mathrm{A}$. The IR spectra were recorded with an infrared spectrometer (Perkin Elmer's System 2000) using the KBr pellet method. The bulk elemental composition of the solids was analyzed using an XRF spectrometer (Phillips X'Unique). The inorganic and organic components in the non-calcined solids were studied by a thermogravimetry analyzer 
(Perkin Elmer's TGA 4000, $20^{\circ} \mathrm{C} / \mathrm{min}$ air flow). Nitrogen adsorption-desorption isotherms were obtained at $-196{ }^{\circ} \mathrm{C}$ using a Micromeritics ASAP 2010 apparatus after outgassing the samples at 250 ${ }^{\circ} \mathrm{C}$ under vacuum overnight.

\section{Results and discussion}

\subsection{XRD analysis}

In this study, SAPO-5 samples have been synthesized using three different imidazolium cations, namely, $[\mathrm{pmIm}]^{+},[\mathrm{bzmIm}]^{+}$and $[\mathrm{dhmIm}]^{+}$(Table 1). These cations possesses similar backbone molecular structures but with different functional groups. Consequently, the templates have different molecular sizes, geometries and electronegativities, resulting in different polarities of the imidazolium molecules. In respect to this, Sanderson's model of intermediate electronegativity $\left(\mathrm{S}_{\text {int }}\right)$ is used to describe the polarities of the imidazolium molecules [32]. As anticipated, $[\mathrm{dhmIm}]^{+}$, which containing polar diol group, has the highest $S_{\text {int }}(2.747)$, followed by $[\mathrm{bzmIm}]^{+}\left(\mathrm{S}_{\mathrm{int}}=2.693\right)$ that has better electron donation ability than $[\mathrm{pmIm}]^{+}\left(\mathrm{S}_{\mathrm{int}}=2.605\right)$.

The XRD analysis was used to monitor the crystallization evolution of SAPO-5 samples. It was shown that the most polar $[\mathrm{dhmIm}]^{+}$template enhanced the crystallization rate of SAPO-5. Significant XRD peaks at $2 \theta=7.51^{\circ}, 15.01^{\circ}, 19.92^{\circ}, 21.12^{\circ}, 22.52^{\circ}$ and $26.13^{\circ}$ were detected in dhmIm-0.3 indicating that crystalline AFI framework had started to form in this solid within the first $0.3 \mathrm{~h}$ of heating (Fig. 1c). In contrast, the pmIm and bzmIm solids remained as amorphous at this early stage as no XRD peaks were observed (Fig. 1a, b). The crystallinity of the $[\mathrm{dhmIm}]^{+}$-templated sample increased linearly with time and reached its maximum (100\% crystallinity) within $2.5 \mathrm{~h}$ (Fig. 1d). Further heating the sample, however, promoted co-crystallization of tridymite dense phase (XRD results not presented here). 
On the other hand, the crystallization profiles of both pmIm- $n$ and bzmIm- $n$ samples followed a sigmoid function (Fig. 1d). The bzmIm- $n$ samples had the longest induction time before nucleation and substantial crystallization only occurred after $8 \mathrm{~h}$ of heating (Fig. 1b, d). The bzmIm-10 achieved $87 \%$ of crystallinity after $10 \mathrm{~h}$ of heating, and no competing phase was co-crystallized in this sample. For the pmIm- $n$ series, significant crystallization of SAPO-5 started around $1.3 \mathrm{~h}$ of heating (Fig. 1a), but the samples had the slowest crystallization kinetics as compared with the samples templated by $[\mathrm{dhmIm}]^{+}$and $[\mathrm{bzmIm}]^{+}$(Fig. 1d). After $5 \mathrm{~h}$ of heating, only $68 \%$ of crystallinity was recorded for the bzmIm-5 sample. Of note, six additional XRD signals (marked as *) were observed at high $2 \theta$ range which could be attributed to a periodic crystal-like arrangement of the $[\mathrm{pmIm}]^{+}$molecules within the solid sample (Fig. 1a) [33]. These six XRD peaks, however, disappeared after the template was removed by calcination.

The XRD results suggested that the $[\mathrm{dhmIm}]^{+}$might have promoted the crystallization rate via its diol group where this functional group could enhance the positive charge of the imidazolium ring by withdrawing its electron density. As a result, the imidazolium ring became more polarized and strong templating effect was therefore enhanced [34]. On the other hand, the $\pi$ electrons from the benzyl group of $[\mathrm{bzmIm}]^{+}$were likely to be attracted to the imidazolium ring and thus the imidazolium cation became less polarized. Consequently, the templating power of $[\mathrm{bzmIm}]^{+}$was reduced and causing the bzmIm- $n$ samples experienced the longest induction period before significant crystalline phase was detected by XRD.

\subsection{Solid yield analysis}

Fig. 2 shows the yield of solid products obtained using different templates versus heating time. Before hydrothermal crystallization, the initial precursor mixtures of the three synthesis series (pmIm- 
0, bzmIm-0 and dhmIm-0) gave about 4.0-5.3\% solid yields. During the crystallization process, however, the solid yields varied depending on the type of imidazolium templates used. For example, the sample prepared from the most polar $[\mathrm{dhmIm}]^{+}$template $\left(S_{\mathrm{int}}=2.747\right)$ exhibited fast increment in the product yield where $89.6 \%$ of solid product was obtained after $2.5 \mathrm{~h}$ of hydrothermal crystallization. In contrast, lower solid product yield was gained when less polar $[\mathrm{pmIm}]^{+}(39.6 \%)$ and $[\mathrm{bzmIm}]^{+}(6.2 \%)$ were used after $2.5 \mathrm{~h}$ of hydrothermal crystallization. For pmIm-5 and bzmIm-10, only $68.4 \%$ and $42.7 \%$ of solid products were successfully recovered, respectively. Hence, it can be seen that the degree of crystallinity and the solid yield have strong correlation with the polarity of the imidazolium template.

\subsection{FESEM analysis}

The FESEM micrographs of SAPO-5 prepared using $[\mathrm{pmIm}]^{+},[\mathrm{bzmIm}]^{+}$and $[\mathrm{dhmIm}]^{+}$ templates are shown in Fig. 3. The pmIm-10 crystallites are of a plate-like morphology with a dimension of about $350 \mathrm{~nm}$ wide and $130 \mathrm{~nm}$ thick (Fig. 3a, b). The FESEM data hence agrees well with the XRD results where this sample exhibits broader XRD peaks (Fig. 1a) owing to their submicron crystallite size [35]. Meanwhile, hexagonal prism and cubic-like SAPO-5 crystallites (bzmIm-10) with an average size of $560 \mathrm{~nm}$ are produced when [bzmIm] $]^{+}$template is employed (Fig. 3c, d). On the other hand, the SAPO-5 crystallites (dhmIm-2.5), synthesized with the polar $[\mathrm{dhmIm}]^{+}$, exhibit typical hexagonal prism morphology with sizes up to a few micrometers (Fig. 3e, f). As can be seen, the crystallites have very high tendency to agglomerate forming larger secondary particles with sizes greater than $10 \mu \mathrm{m}$. Hence, the results clearly show that the morphologies of the three SAPO-5 samples are significantly affected by the polarity, functional group and geometry of the imidazolium templates used. 


\subsection{Elemental analysis}

The effect of various imidazolium templates used on the final chemical composition (P:Al:Si ratio) of the SAPO-5 samples (pmIm-5, bzmIm-10 and dhmIm-2.5) was also studied (Table 2). The data show that dhmIm-2.5 experiences the highest degree of isomorphous incorporation of $\mathrm{Si}$ into the AFI aluminophosphate framework $(\mathrm{Si} /(\mathrm{P}+\mathrm{Al}+\mathrm{Si})=0.068)$ while $\mathrm{pmIm}-5$ has the least $\mathrm{Si}$ incorporated among the three samples $(\mathrm{Si} /(\mathrm{P}+\mathrm{Al}+\mathrm{Si})=0.054)$. Thus, the results of the elemental analysis reveal that the polar $[\mathrm{dhmIm}]^{+}$template tend to attract silicate anionic species strongly, and hence indirectly promoting the participation and isomorphous substitution of Si into the formation of microporous AFI framework.

\subsection{TG/DTG measurements}

The chemical and supramolecular interactions of the imidazolium templates with the microporous solids were studied via thermogravimetry analysis. The thermogravimetry data of the assynthesized SAPO-5 are summarized in Table 3. In addition, the TGA/DTG profiles of free [pmIm] $\mathrm{PH}$, [bzmIm] OH and [dhmIm]OH templates are also shown in Fig. 4 whereas the TGA/DTG profiles of the as-synthesized pmIm-5, bzmIm-10 and dhmIm-2.5 samples are shown in Fig. 5. As can be seen, those free imidazolium hydroxide templates exhibit two steps of weight loss (Fig. 4). The first endotherm below $220{ }^{\circ} \mathrm{C}$ is due to the adsorbed water ([pmIm]OH: $3.59 \%$, [bzmIm]OH: $4.48 \%$, [dhmIm]OH: $19.41 \%$ ) while the second step of weight loss around $210-480{ }^{\circ} \mathrm{C}$ is attributed to the decomposition of the imidazolium hydroxides ([pmIm]OH: 96.40\%, [bzmIm] OH: 95.52\%, [dhmIm]OH: 60.88\%). The thermogravimetry data show that $[\mathrm{pmIm}] \mathrm{OH}$ and $[\mathrm{bzmIm}] \mathrm{OH}$ have completely combusted at $385{ }^{\circ} \mathrm{C}$ and $383{ }^{\circ} \mathrm{C}$, respectively. In contrast, the relatively polar [dhmIm] OH molecules, which contain diol 
groups, require higher temperature $\left(496^{\circ} \mathrm{C}\right)$ for partial decomposition, leaving $19.79 \%$ of noncombusted carbon even after $800{ }^{\circ} \mathrm{C}$.

When the imidazolium templates are confined within the solids, the as-synthesized SAPO-5 samples show different weight loss regions with multiple steps of thermal decomposition (Fig. 5). For example, a slight difference is shown in the first step of weight loss at $210^{\circ} \mathrm{C}$ by the pmIm-5, bzmIm10 and dhmIm-2.5 samples $(4.76 \%, 3.85 \%$ and $1.21 \%$, respectively). The amount of water adsorbed is inversely proportional to the hydrophilicity/polarity of imidazolium templates employed where dhmIm-2.5 which containing polar $[\mathrm{dhmIm}]^{+}$template adsorbs the least amount of water. Besides, the amount of template occluded in the micropores of SAPO-5 is also different. The bzmIm-10 has the lowest amount of template trapped in the pores $(9.47 \%$, see Table 3$)$ due to its large molecular size which allows its limited accommodation in the micropores, whereas dhmIm-2.5 has the largest amount of confined template (12.90\%), followed by pmIm-5 (10.77\%). As a result, dhmIm-2.5 adsorbs lesser water than pmIm-5 and bzmIm-10. Moreover, the cationic template molecules, which are confined in the porous framework, also experience delayed thermal decomposition. For example, full decomposition of $[\mathrm{pmIm}]^{+}$within SAPO-5 is observed at $515{ }^{\circ} \mathrm{C}$, and it is $128{ }^{\circ} \mathrm{C}$ higher than that of the free $[\mathrm{pmIm}]^{+}$template $\left(385^{\circ} \mathrm{C}\right)$. For bzmIm-10 and dhmIm-2.5, similar trend is also observed where the confined $[\mathrm{bzmIm}]^{+}$and $[\mathrm{dhmIm}]^{+}$decompose completely at higher temperatures $\left(590{ }^{\circ} \mathrm{C}\right.$ and $710^{\circ} \mathrm{C}$, respectively) in respect to their free counterpart $\left(383{ }^{\circ} \mathrm{C}\right.$ and $496^{\circ} \mathrm{C}$, respectively). Among the three as-synthesized SAPO-5 samples, dhmIm-2.5 requires the highest temperature to remove fully the occluded organic template. This can be explained by (i) the strong intra-/intermolecular hydrogen bonding between the $[\mathrm{dhmIm}]^{+}$and the SAPO-5 framework, (ii) the supramolecular/confinement effect of the rigid microporous solid, and (iii) the formation of covalent bond via condensation reaction between the $-\mathrm{OH}$ groups of $[\mathrm{dhmIm}]^{+}$with the defect sites $(\mathrm{Al}-\mathrm{OH}, \mathrm{P}-\mathrm{OH}, \mathrm{Si}-\mathrm{OH})$ of $\mathrm{SAPO}-5$ in the microporous channels [36, 37]. 


\section{6. $\quad$ Surface and texture properties}

The properties of imidazolium templates not only affect the crystallization process and the morphology but also govern the porous properties of the resulted SAPO-5 samples. The nitrogen gas adsorption and desorption isotherms of the calcined SAPO-5 samples are shown in Fig. 6. All the samples (pmIm-5, bzmIm-10 and dhmIm-2.5) display Type I isotherm, which is characteristic of microporous materials [38]. Typically, the isotherms rise sharply and reach a plateau at low relative pressure $\left(\mathrm{P} / \mathrm{P}_{\mathrm{o}}\right)$. At higher relative pressure $\left(\mathrm{P} / \mathrm{P}_{\mathrm{o}}>0.4\right)$, further nitrogen uptake with hysteresis loops is observed for the pmIm-5 and bzmIm-10 samples (Fig. 6). This H3 type-like hysteresis loop can be related to slit-shaped mesoporosity that is usually formed between submicron-sized particles [38]. However, no hysteresis loop is shown by the dhmIm-2.5 isotherm at high relative pressure. Instead, it shows an abrupt slight increase in nitrogen uptake at $\mathrm{P} / \mathrm{P}_{\mathrm{o}}>0.90$, which can be explained by the presence of small volume of macroporosity generated from close packing of the micron-sized SAPO5 crystallites.

The dhmIm-2.5 exhibits the lowest microporosity $\left(S_{\text {mic }}=181 \mathrm{~m}^{2} / \mathrm{g}\right.$, see Table 2$)$ due to partial collapse of the AFI microporous framework as a result of severe calcination temperature $\left(800{ }^{\circ} \mathrm{C}\right)$ needed to remove completely the strongly occluded $[\mathrm{dhmIm}]^{+}$. In contrast, both pmIm-5 and bzmIm10 have almost similar microporosity $\left(\mathrm{S}_{\mathrm{mic}} \sim 260 \mathrm{~m}^{2} / \mathrm{g}\right)$ as $[\mathrm{pmIm}]^{+}$and $[\mathrm{bzmIm}]^{+}$are much easier to be decomposed and removed from the microporous channels (calcined at 550 and $600{ }^{\circ} \mathrm{C}$, respectively) due to their weaker interactions and occlusion in the SAPO-5 crystalline framework. The data in Table 2 show that the pmIm-5 sample has the highest specific BET surface area and pore volume $\left(269 \mathrm{~m}^{2} / \mathrm{g}\right.$, $\left.0.39 \mathrm{~cm}^{3} / \mathrm{g}\right)$, followed by bzmIm-10 $\left(261 \mathrm{~m}^{2} / \mathrm{g}, 0.35 \mathrm{~cm}^{3} / \mathrm{g}\right)$ and $\mathrm{dhmIm}-2.5\left(182 \mathrm{~m}^{2} / \mathrm{g}, 0.30 \mathrm{~cm}^{3} / \mathrm{g}\right)$. Thus, the results indicate that smaller and less polar organic templates are more preferable in 
crystallizing SAPO-5 with larger surface area and pore volume as they are loosely confined and weakly interacted in the SAPO- $n$ frameworks.

\section{Conclusions}

As a conclusion, the effects of imidazolium templates with various functional groups in the crystallization of microporous SAPO-5 have been investigated. The results show that the polarity and molecular geometry of imidazolium templates govern several physico-chemical properties of the resulted SAPO-5. By using polar template such as $[\mathrm{dhmIm}]^{+}$, the crystallization rate and solid yield of SAPO-5 can be significantly enhanced. While the chemical composition is slightly affected upon changing the templates, a noteworthy change in the morphology of SAPO-5 is achieved. Different degree of template occlusion by crystalline microporous AFI framework has been observed where $[\mathrm{dhmIm}]^{+}$with polar diol functional group interacts very strongly with the inorganic host, while $[\mathrm{pmIm}]^{+}$with simple linear propyl chain is loosely bound to the framework. As a result, $[\mathrm{pmIm}]^{+}$can be easily removed from the sub-micron crystallites using lower temperature calcination, producing SAPO-5 solids with the highest surface area, pore volume and microporosity. Hence, this study demonstrates that the selection of organic templates with proper geometry and contain hydrophobic functional groups is utmost important to produce SAPO- $n$ with high microporosity and surface area.

\section{Acknowledgments}

The financial support from FRGS (203/PKIMIA/6711642) grant is gratefully acknowledged. I.A. Auwal would also like to thank the USM Fellowship and TETFund for the scholarship provided.

\section{References}


[1] S. Henninger, F. Schmidt, H.-M. Henning, Appl. Therm. Eng. 30 (2010) 1692-1702.

[2] L. Tosheva, E.P. Ng, S. Mintova, M. Hölzl, T.H. Metzger, A.M. Doyle, Chem. Mater. 20 (2008) 5721-5726.

[3] E.-P. Ng, L. Delmotte, S. Mintova, Green Chem. 10 (2008) 1043-1048.

[4] N. Sheng, Y. Chu, S. Xin, Q. Wang, X. Liu, J. Xu, F.-S. Xiao, F. Deng, Inorg. Chem. Front. 5 (2018) 1633-1639.

[5] C.-M. Wang, R.Y. Brogaard, Z.-K. Xie, F. Studt, Catal. Sci. Technol. 5 (2015) 2814-2820.

[6] L. Bacakova, M. Vandrovcova, I. Kopova, I. Jirka, Biomater. Sci. 6 (2018) 974-989.

[7] D.Y. Khoo, W.-M. Kok, R.R. Mukti, S. Mintova, E.-P. Ng, Solid State Sci. 25 (2013) 63-69.

[8] X. Tong, J. Xu, C. Wang, W. Yan, J. Yu, F. Deng, R. Xu, Micropor. Mesopor. Mater. 183 (2014) 108-116.

[9] D.Y. Khoo, H. Awala, S. Mintova, E.-P. Ng, Micropor. Mesopor. Mater. 194 (2014) 200-207.

[10] C.S. Cundy, P.A. Cox, Micropor. Mesopor. Mater. 82 (2005) 1-78.

[11] X. Zhao, J. Zhao, C.-Y. Chiang, Z. Li, Y. Zhao, W. Zhou, New J. Chem. 40 (2016) 24442450 .

[12] S. Brace, P. Wormald, R.J. Darton, Phys. Chem. Chem. Phys. 17 (2015) 11950-11953.

[13] B. Boonchom, S. Youngme, T. Srithanratana, C. Danvirutai, J. Therm. Anal. Calorim. 91 (2008) 511-516.

[14] N. Rajic, N.Z. Logar, A. Golobic, V. Kaucic, J. Phys. Chem. Solid. 64 (2003) 1097-1103.

[15] M.M. Azim, A. Stark, Micropor. Mesopor. Mater. 272 (2018) 251-259.

[16] H. Bahrami, J.T. Darian, M. Sedighi, Micropor. Mesopor. Mater. 261 (2018) 111-118.

[17] A.I. Benin, S.-J. Hwang, S.I. Zones, D. Xie, K. Chaudhuri, H. Lacheen, Micropor. Mesopor. Mater. 274 (2019) 257-265.

[18] E.-P. Ng, D.T.-L. Ng, H. Awala, K.-L. Wong, S. Mintova, Mater. Lett. 132 (2014) 126-129.

[19] M.M. Azim, A. Pensado, B. Kirchner, T. Gutmann, P.B. Groszewicz, G. Buntkowsky, A. Stark, Micropor. Mesopor. Mater. 266 (2018) 204-213.

[20] IZA-SC Database of Zeolite Structures. http://www.iza-structure.org/ databases/.

[21] D.-G. Cheng, F. Chen, X. Zhan, Appl. Catal. A: Gen. 435 (2012) 27-31.

[22] E.-P. Ng, H. Awala, S. Komaty, S. Mintova, Micropor. Mesopor. Mater. 280 (2019) 256-263.

[23] Ö. Weiß, G. Ihlein, F. Schüth, Micropor. Mesopor. Mater. 35 (2000) 617-620.

[24] P. Rungrojchaipon, X. Wang, A.J. Jacobson, Micropor. Mesopor. Mater. 109 (2008) 478-484. 
[25] E.-P. Ng, K.-L. Wong, D.T.-L. Ng, H. Awala, R.R. Mukti, F. Adam, S. Mintova, Mater. Chem. Phys. 188 (2017) 49-57.

[26] S. Mintova, S. Mo, T. Bein, Chem. Mater. 10 (1998) 4030-4036.

[27] S.H. Jhung, J.-S. Chang, Y.K. Hwang, S.-E. Park, J. Mater. Chem. 14 (2004) 280-285.

[28] F. Jiang, J. Zhai, J. Ye, J. Han, Z. Tang, J. Cryst. Growth 283 (2005) 108-114.

[29] C. Zhao, Y. Yang, W. Chen, H. Wang, D. Zhao, P.A. Webley, CrystEngComm 11 (2009) 739-742.

[30] M. Li, C. Zeng, L. Zhang, CrystEngComm 14 (2012) 3787-3792.

[31] E.-P. Ng, J.-P. Ghoy, H. Awala, A. Vicente, R. Adnan, T.C. Ling, S. Mintova, CrystEngComm 18 (2016) 257-265.

[32] R.T. Sanderson, J. Am. Chem. Soc. 105 (1983) 2259-2261.

[33] J.C. Manayil, A.F. Lee, K. Wilson, Molecules 24 (2019) 239.

[34] M.M. Azim, A. Stark, ChemistrySelect 3 (2018) 12495-12503.

[35] E.-P. Ng, G.K. Lim, G.-L. Khoo, K.H. Tan, B.S. Ooi, F. Adam, T.C. Ling, K.-L. Wong, Mater. Chem. Phys. 155 (2015) 30-35.

[36] E.-P. Ng, S.S. Sekhon, S. Mintova, Chem. Commun. 0 (2009) 1661-1663.

[37] M. Sánchez, R.D. Díaz, T. Córdova, G. González, F. Ruette, Micropor. Mesopor. Mater. 203 (2015) 91-99.

[38] K.S.W. Sing, D.H. Everett, R.A.W. Haul, L. Moscou,R.A. Pierotti, J. Rouquerol, T. Siemieniewska, Pure Appl.Chem. 57 (1985) 603-619. 


\section{$\underline{\text { Figures captions }}$}

Fig. 1. XRD patterns of (a) pmIm- $n$, (b) bzmIm- $n$ and (c) dhmIm- $n$ solids after various heating times, and (d) a plot of crystallinity versus respective crystallization heating time of pmIm- $n$, bzmIm$n$ and dhmIm- $n$ samples. * shows the XRD diffraction peaks due to the periodic crystal-like arrangement of $[\mathrm{pmIm}]^{+}$molecules in the SAPO-5 micropores.

Fig. 2. Solid yield profiles of (a) pmIm- $n$, (b) bzmIm- $n$ and (c) dhmIm- $n$ samples.

Fig. 3. FESEM images of (a,b) pmIm-5, (c,d) bzmIm-10 and (e,f) dhmIm-2.5 SAPO-5 crystals under different magnifications.

Fig. 4. TGA-DTG profiles of (a) [pmIm]OH, (b) [bzmIm]OH and (c) [dhmIm]OH.

Fig. 5. TGA-DTG profiles of as-synthesized (a) pmIm-5, (b) bzmIm-10 and (c) dhmIm-2.5 SAPO-5.

Fig. 6. Nitrogen gas adsorption and desorption isotherms of calcined (a) pmIm-5, (b) bzmIm-10 and (c) dhmIm-2.5 SAPO-5. 
$\underline{\text { Figures }}$
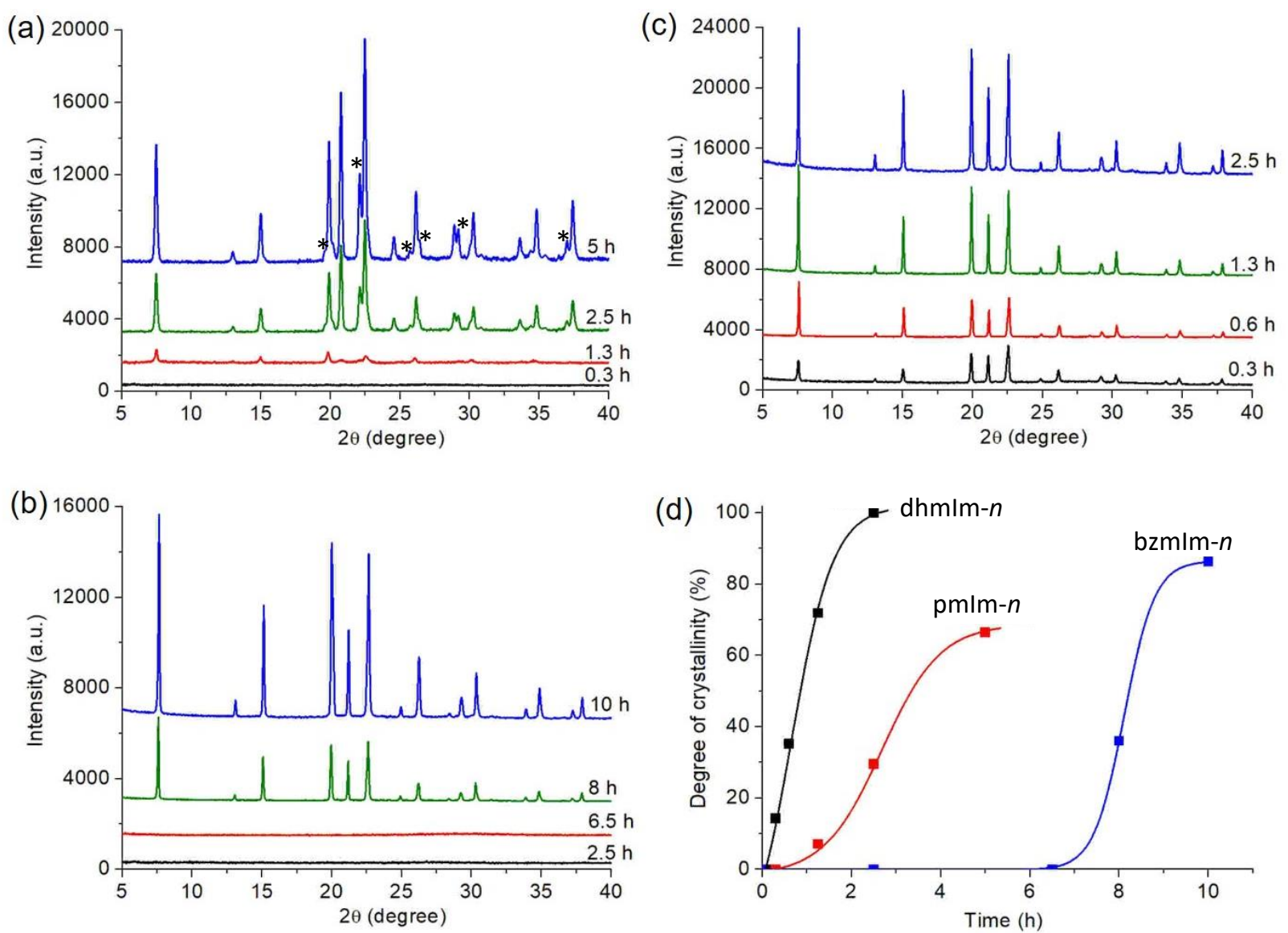

Fig. 1. 


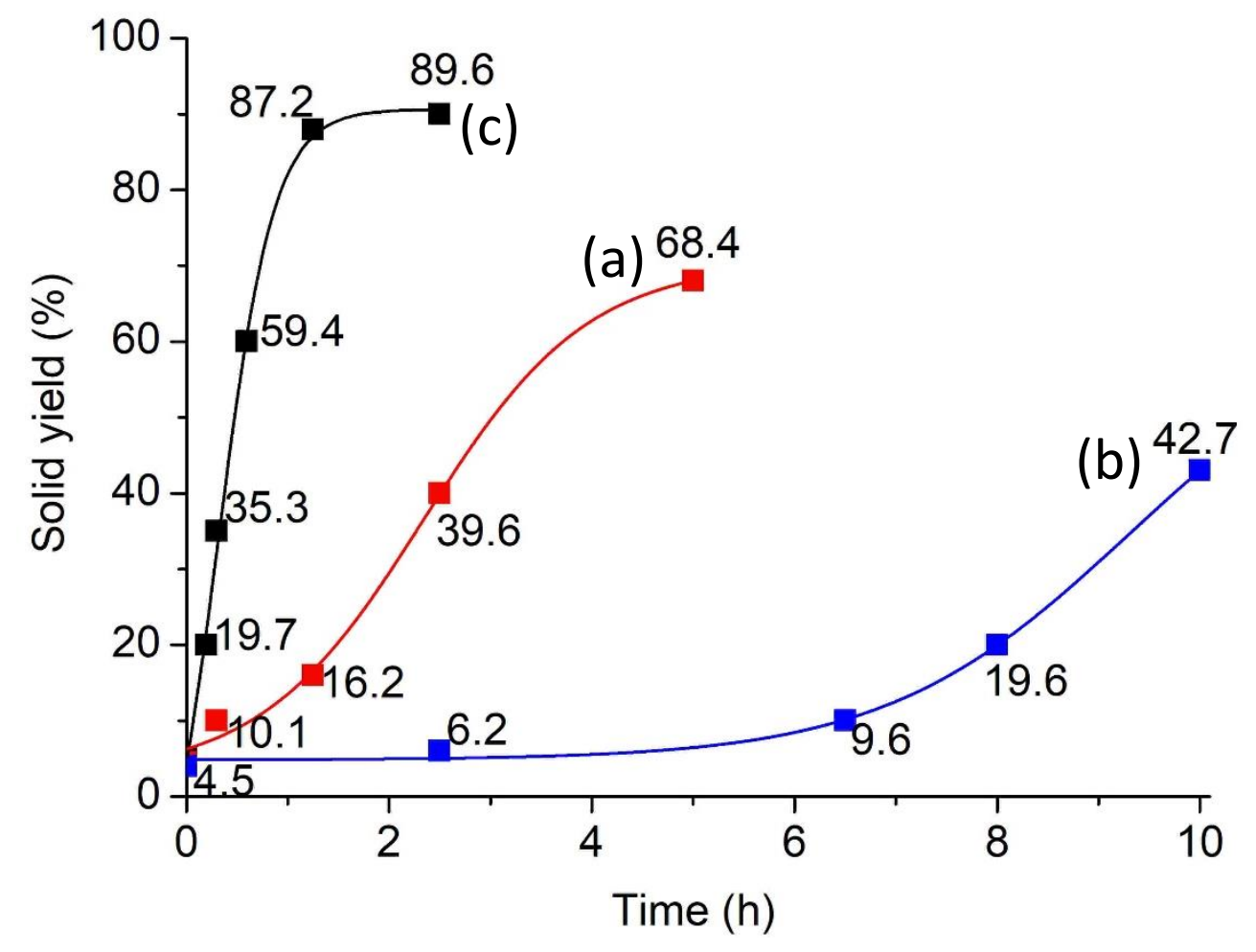

Fig 2. 


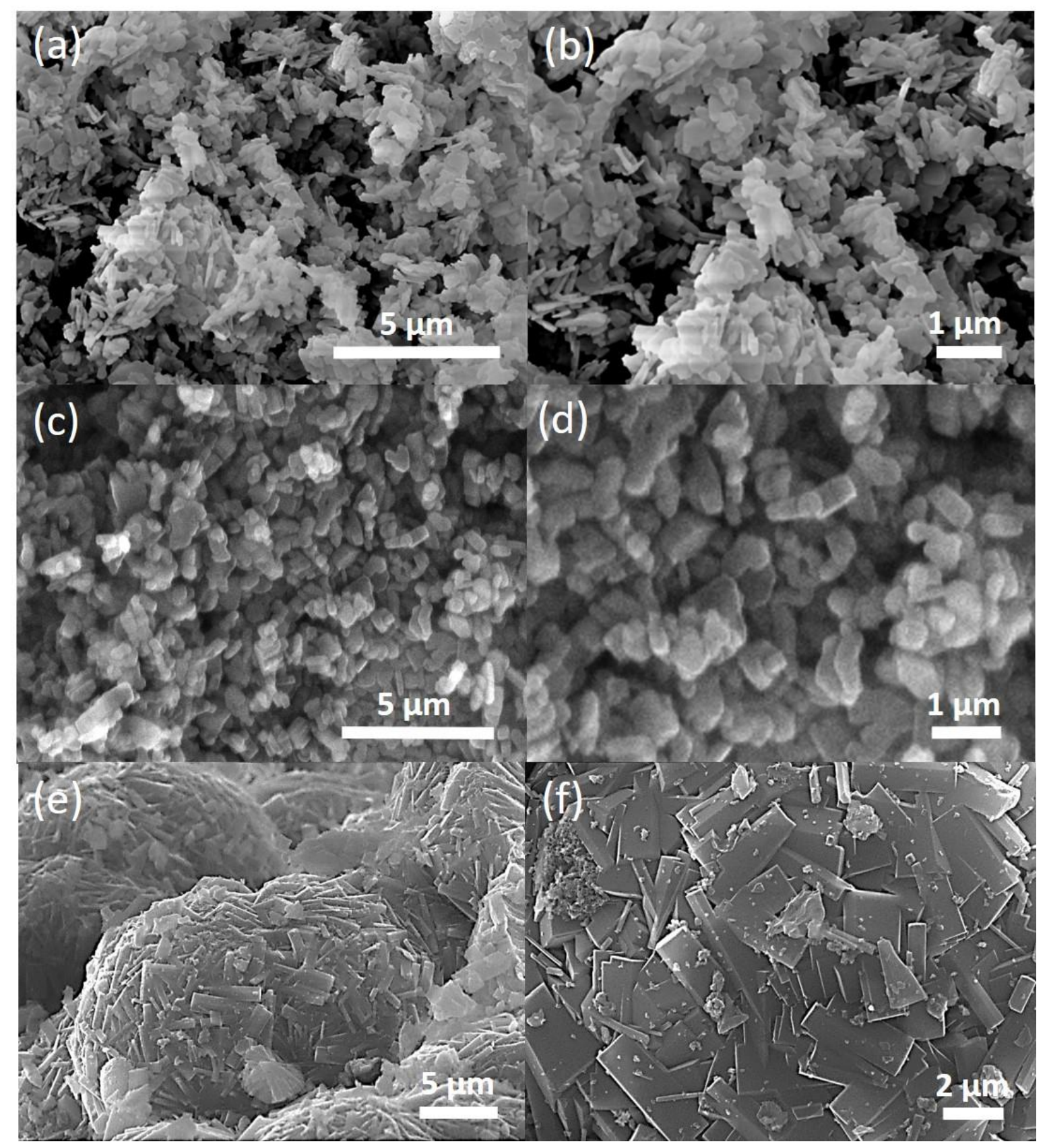

Fig. 3. 

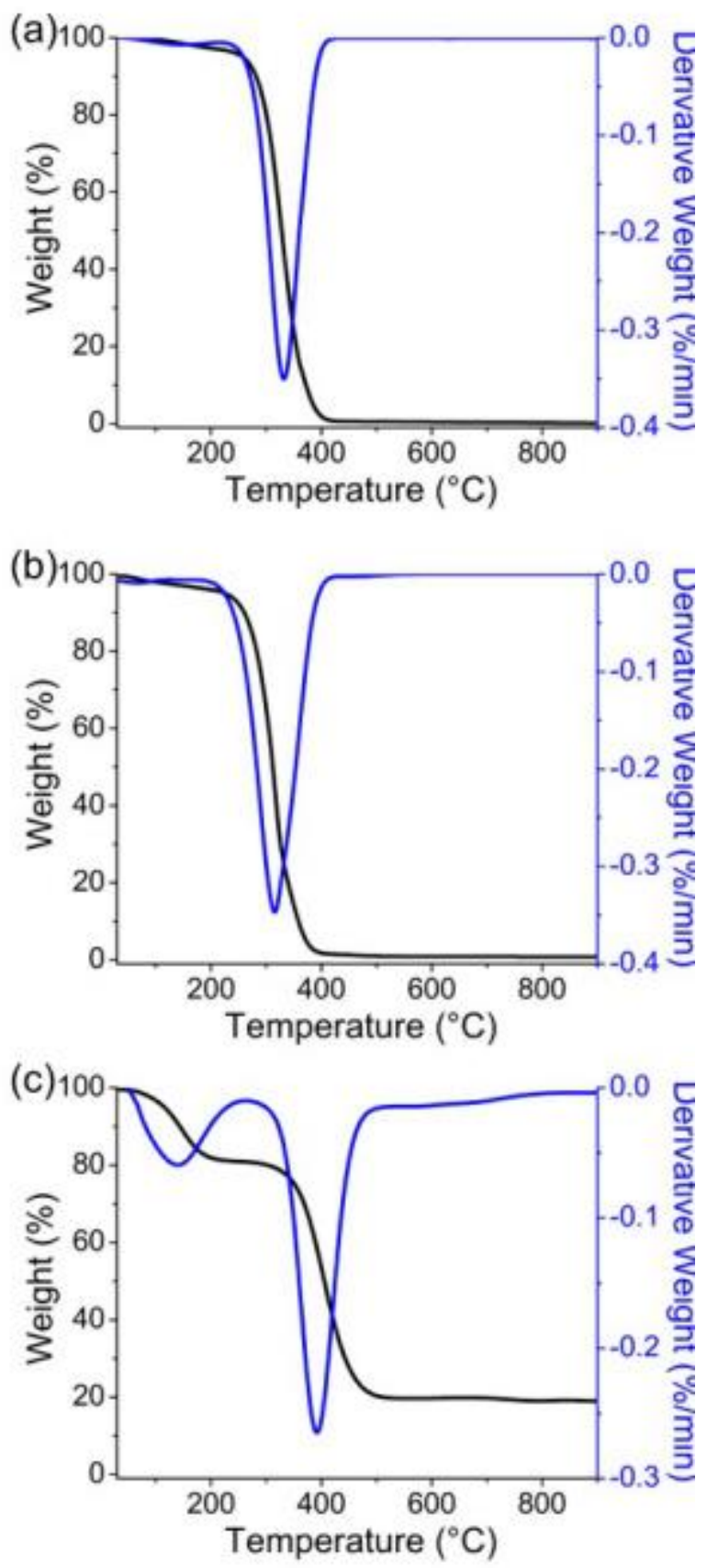

Fig. 4. 

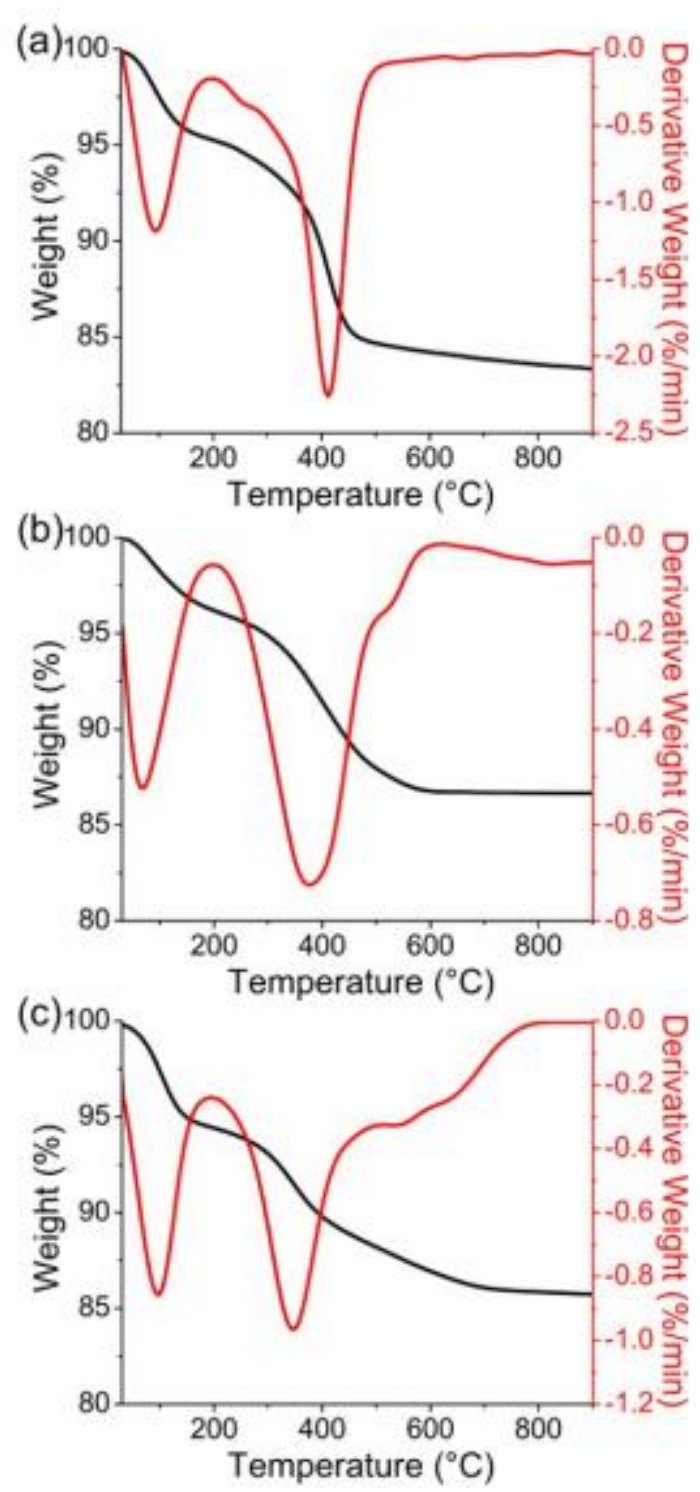

Fig. 5. 


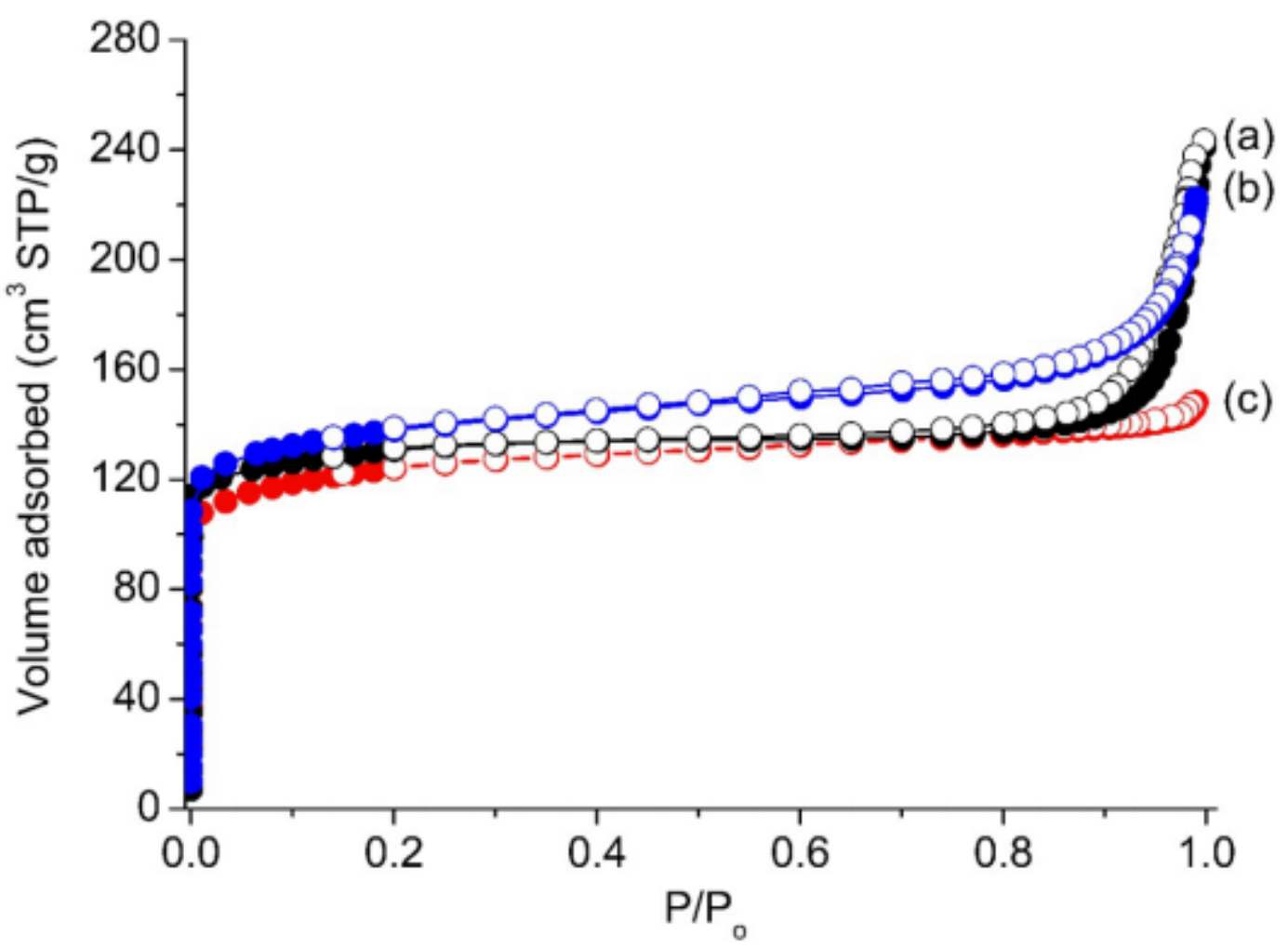

Fig. 6. 


\section{$\underline{\text { Table captions }}$}

Table 1. General information of imidazolium cations used in this study.

Table 2. Chemical composition and physicochemical properties of SAPO-5 solids.

Table 3. Thermogravimetry data of as-synthesized SAPO-5 solids. 


\section{TABLES}

Table 1

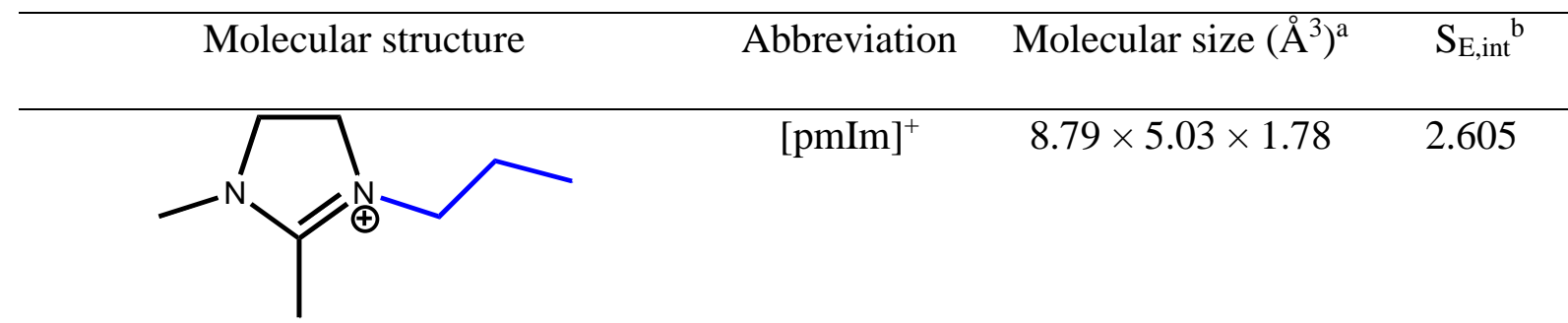

1-Propyl-2,3-dimethyl-1H-imidazol-3-ium

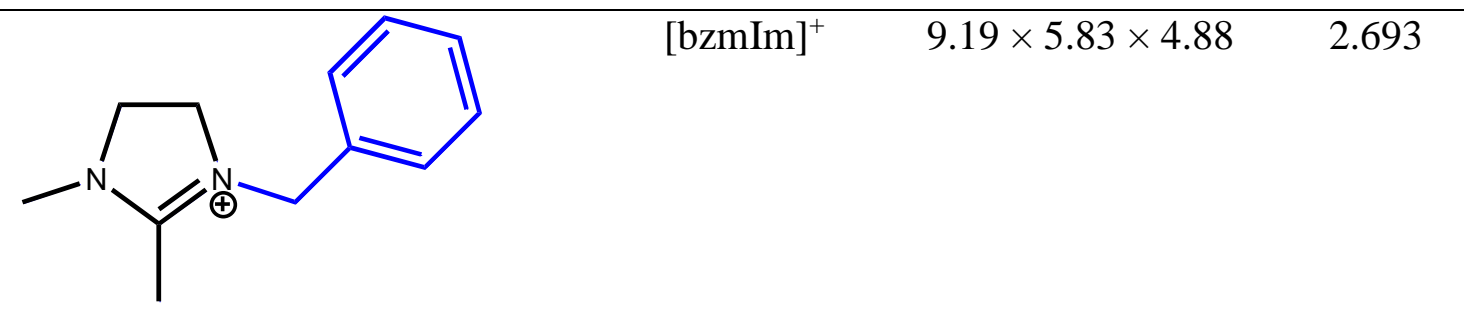

1-Benzyl-2,3-dimethyl-1H-imidazol-3-ium

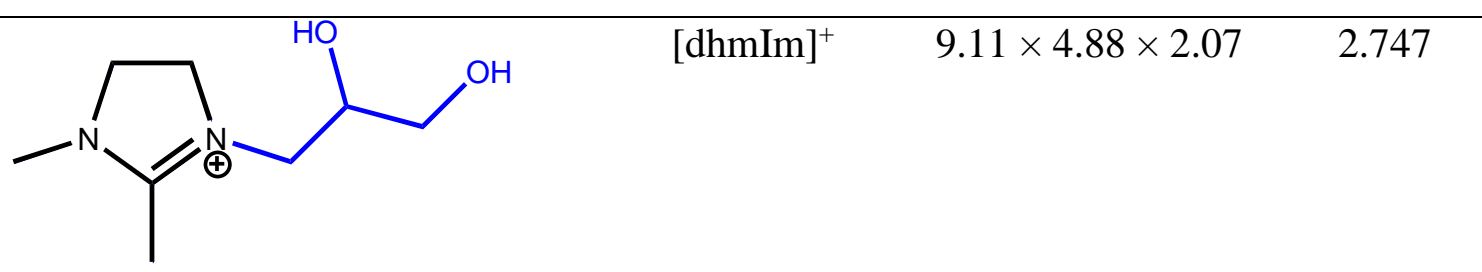

3-(2,3-dihydroxypropyl)-1,2-dimethyl-

1H-imidazol-3-ium

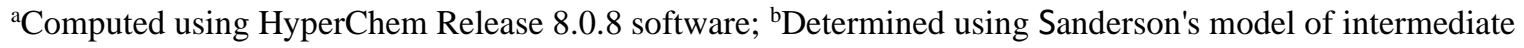
electronegativity, $S_{\mathrm{E}, \mathrm{int}}=\left(\Pi \mathrm{S}_{\mathrm{E}, \mathrm{Z}}^{\mathrm{x}}\right)^{(\Sigma \mathrm{x})^{-1}}$ 
Table 2

\begin{tabular}{lccccccc}
\hline Samples & $\begin{array}{c}\mathrm{P} / \mathrm{Al} \\
\mathrm{ratio}^{\mathrm{a}}\end{array}$ & $\begin{array}{c}\mathrm{Si} / \mathrm{Al} \\
\mathrm{ratio}^{\mathrm{a}}\end{array}$ & $\begin{array}{c}\mathrm{Si} /(\mathrm{P}+\mathrm{Al}+\mathrm{Si}) \\
\mathrm{ratio}^{\mathrm{a}}\end{array}$ & $\begin{array}{c}\mathrm{S}_{\mathrm{BET}}{ }^{\mathrm{b}} \\
\left(\mathrm{m}^{2} / \mathrm{g}\right)\end{array}$ & $\begin{array}{c}\mathrm{S}_{\mathrm{ext}}{ }^{\mathrm{c}} \\
\left(\mathrm{m}^{2} / \mathrm{g}\right)\end{array}$ & $\begin{array}{c}\mathrm{S}_{\mathrm{mic}^{\mathrm{d}}} \\
\left(\mathrm{m}^{2} / \mathrm{g}\right)\end{array}$ & $\begin{array}{c}\mathrm{V}_{\mathrm{tot}^{\mathrm{e}}} \\
\left(\mathrm{cm}^{3} / \mathrm{g}\right)\end{array}$ \\
\hline pmIm-5 & 0.85 & 0.105 & 0.054 & 269 & 23 & 246 & 0.39 \\
bzmIm-10 & 0.87 & 0.105 & 0.056 & 261 & 11 & 250 & 0.35 \\
dhmIm-2.5 & 0.83 & 0.134 & 0.068 & 182 & 1 & 181 & 0.30 \\
\hline
\end{tabular}

a Measured by ICP-OES; ${ }^{b}$ Specific BET surface area; ${ }^{b}$ External surface area; ${ }^{c}$ Micropore surface area;

${ }^{\mathrm{d}}$ Total pore volume.

Table 3

\begin{tabular}{cccc}
\hline $\begin{array}{c}\text { As-synthesized } \\
\text { SAPO-5 samples }\end{array}$ & \multicolumn{2}{c}{ Weight loss $(\%)$} & \multirow{2}{*}{$\begin{array}{c}\text { Weight of inorganic } \\
\text { residue }(\%)\end{array}$} \\
\cline { 2 - 3 } pmIm-5 & $4200^{\circ} \mathrm{C}$ & $200^{\circ} \mathrm{C}$ to $x^{\circ} \mathrm{C}$ & 84.47 \\
bzmIm-10 & 4.76 & $10.77(x=520)$ & 86.68 \\
dhmIm-2.5 & 3.85 & $9.47(x=600)$ & 85.89 \\
\hline \hline
\end{tabular}




\section{$\underline{\text { Research Highlights }}$}

- SAPO-5 is synthesized using imidazolium-based templates.

- Template's functional group affects crystallization and morphological profiles.

- Proper hydrophobicity is essential to control host-guest chemical interactions.

- Polar template interacts strongly with SAPO-5, hence high temperature calcination is needed.

\section{$\underline{\text { Graphical abstract }}$}

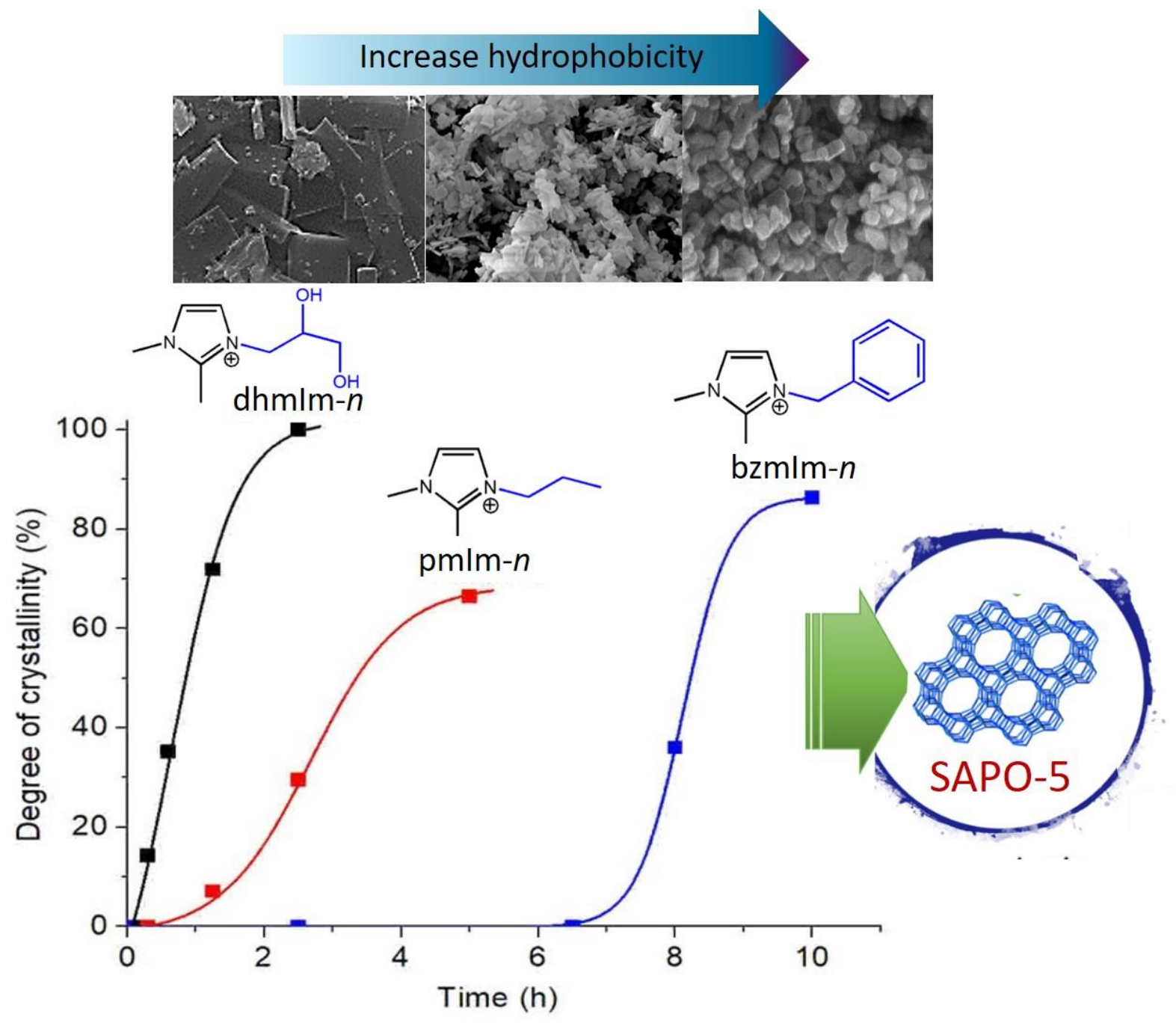


